\title{
Colour control and selectivity in TiAIN solar-thermal absorbers
}

Shuxi Zhao ${ }^{\mathrm{a}}$, Dechun Zhu ${ }^{\mathrm{b}}$ and Carl-G.Ribbing ${ }^{\mathrm{a}}$

a) Div. of Solid State Physics, Ångström Laboratory, Uppsala University

Box 534, SE-751 21 Uppsala, Sweden

b) Dept. of Chemical and Materials Engineering, Hefei University

230022, Hefei, PR. China

\begin{abstract}
Optical constants for simulations were obtained by R- and T-measurements on TiAlN thin films deposited on Corning 7059 glass. The model parameterized free carrier effects and an inter-band excitation. The calculations demonstrated that the colour effects are due to interference and inter-band absorption around $500 \mathrm{~nm}$ in a single layer coating. The peak shifts with the thickness of the thin film which gives a simple way to obtain different colours. Solar absorptance of $86 \%$ can be reached already for a single TiAlN-film on an Al substrate.
\end{abstract}

Keywords: Solar absorbers, selectivity, colour, Brendel oscillators.

\section{INTRODUCTION}

Our work concerns the trade-off between two properties of thermal solar collectors based on a titanium aluminium nitride surface. The first one is the "selectivity", i.e. the ability of the absorbing surface to absorb as much as possible of the incoming short-wavelength solar energy and simultaneously avoid losses by thermal emission to the surroundings ${ }^{1}$. This property is quantified by two dimensionless parameters: $\alpha$, the fraction of impinging solar radiation that is absorbed, and $\varepsilon$, the thermal emittance. Typically $\alpha>0.9$ and $\varepsilon<0.1$ is strived for and obtained, at least for fresh surfaces. The second property is the visual colour of the absorber. Considering that solar thermal absorbers most often are planned to be incorporated in the built environment, the visual colour has an important aesthetical function that cannot be neglected in planning large area installations. Colour is specified by two independent CIE colour coordinates ${ }^{2}$.

Considering the selectivity alone, it is obvious that the surface should be black, since the visible part of the spectrum: $0.4-0.8 \mu \mathrm{m}$, is a part of the total incoming solar radiation in the range: $0.3-2.5 \mu \mathrm{m}$.A large area of totally black absorbers may not be aesthetically acceptable. Previous reports from our laboratories have demonstrated that reactive, magnetron sputtering from one or two targets can be used to obtain a wide variety of surfaces, at least in lab-scale, with favourable optical, thermal and mechanical properties for solar absorbers. ${ }^{3,4}$

Advances in Optical Thin Films IV, edited by Michel Lequime, H. Angus Macleod, Detlev Ristau,

Proc. of SPIE Vol. 8168, 81680G · @ 2011 SPIE · CCC code: 0277-786X/11/\$18

doi: $10.1117 / 12.896591$

Proc. of SPIE Vol. 8168 81680G-1 
In particular a surface based on sputtered TiAION on an Al substrate may exhibit several non-black colours with very modest loss in thermal efficiency. Strong dark colours, different from black, cause only weak reflectance losses in the visible. The possibility of close colour control with the same set of sputtering conditions and only adjusting the film thickness was noted empirically by spectral measurements and calculation of the CIE colour coordinates. ${ }^{3,4}$

In this contribution we shall look more closely on the explanation for this observation. The fact that a material with strong absorption caused by interband transitions exhibits colour change in reflectance with thickness will be illustrated with calculations for a single film on a metal substrate. The mechanism is related with, but not identical to that of interference in dielectric films. Introducing now the issues of materials, we start with TiN. It is well-known as a hard compound with important technical use as a wear-protective coating on cutting and grinding tools. It has a golden colour which is the reason for the use as gold protection and replacement. The basic physical reason for this colour is a background for this work. TiN has an excellent metallic electrical conductivity like gold, but the high infrared reflectivity and the golden colour have physically different reasons. Gold is a nearly-free-electron-like metal and the high, almost wavelength independent reflectance for wavelengths $>0.5 \mu \mathrm{m}$ is an example of Drude behaviour in the relaxation region ${ }^{5}$. It is caused by intraband excitation of the nearly free s-electrons of gold. The reflectance step at $0.5 \mu \mathrm{m}$ corresponds to the onset of interband transitions from the d-bands to the the Fermi-level. In contrast, the band-structure of TiN is not freeelectron-like. The Fermi-level in TiN intersects the d-bands, and yet an interval in long-wave VIS and NIR with high, and almost constant reflectance is seen.

The reason for the absence of interband transitions between the d-bands in transition metal nitrides was discussed by Karlsson in $1981^{6}$. It is striking that the corresponding carbides: $\mathrm{TiC}, \mathrm{ZrC}$ and HfC, have no such intervals of high, wavelength-independent reflectance, and they visually appear like transition metals. A qualitative explanation was given by combining the results of APW band-structure calculations ${ }^{7}$, with atomic selection rules that only permits transitions $\Delta \mathrm{l}= \pm \underline{+1}$, i.e. $\mathrm{s} \leftrightarrow \mathrm{p}$ and $\mathrm{p} \leftrightarrow \mathrm{d}$, but not $\mathrm{d} \leftrightarrow \mathrm{d}$ and $\mathrm{s} \leftrightarrow \mathrm{d}$. The LCAO partial density-of-states show that the $\mathrm{p}$ - and d-densities at $\mathrm{E}_{\mathrm{F}}$ are equal in the carbides, while the d-density completely dominates in the nitrides. The selection-rule prohibits $d \leftrightarrow d$ transitions at the Fermi-level and introduces a threshold for interband transitions in the nitrides. The $\mathrm{p} \rightarrow \mathrm{d}$ transitions only occur for $\mathrm{h} v \geq 0.12 \mathrm{Ry}$, i.e. $1.6 \mathrm{eV}$, in agreement with the steep drop in reflectance for the nitrides in the visible spectral range. Later, calculations using the LMTO-method gave similar results and confirmed the difference between the carbides and the nitrides. The authors mention "For the nitrides and oxides, the energy difference between the Fermi level and the p-group of bands has to be overcome for the interband transitions to start." ${ }^{8}$ In this case the matrix elements were explicitly calculated, an effort that replaced the questionable use of a free atom selection rule. 
The above description of appearance of colour in stoichiometric compounds with strong, hybridized atomic orbitals provide a suitable background when shifting to more complex and even non-stoichiometric compounds. Our example is TiAlN. The general interest for this material grew out of the need for more wear-resistant, tribological coatings. It was noted that this compound is as hard and wear-resistant as TiN and has higher oxidation resistance thanks to the formation of a protective surface oxide layer. ${ }^{9}$ The potential optical usefulness of the redshift of the reflectance edge compared with TiN was investigated. The application in this case was for radiative temperature control of a space satellite. The reduced steepness and the redshift of the reflectance edge give lower solar absorptance, as well as higher thermal emittance, i.e. a lower equilibrium temperature, to a satellite exposed to solar radiation, but free to radiate towards space. ${ }^{10}$ At a later stage it was realized that the possibility to control the colour of TiAlN-coatings in a two target sputtering process opened up for the deposition of coloured, selective solar absorbers as mentioned above. ${ }^{3}$ The interband transitions in the mother compound TiN were modified so as to give a modest reflectance drop in the visible range. It was demonstrated that a low average visible reflectance "dark" surface can be modulated with controlled variations giving it a strong, dark colour and yet maintaining a high solar selectivity. Furthermore, by adding a small amount of oxygen to the sputtering atmosphere, an antireflecting TiAlON-coating can be deposited that further enhances the solar selectivity. ${ }^{4}$

In this work we shall report model calculations for the system TiAlN/Al to confirm that different reflected colours can be obtained by sputtering the same inhomogenous material but with different thicknesses. With separate studies of the different films on a transmitting substrate, the parameter values for a dispersive model have been determined and used for thin film calculations.

\section{FORMULATION AND USE OF THE MODELS}

The optical constants of thin film materials are often different from the optical constants of the corresponding bulk materials. Our work requires knowledge of the film as deposited by sputtering, which sometimes results in an inhomogenous structure. In our study we have therefore used two models of dielectric functions. The two models used are simple, well-known versions for a transition metal and a dielectric, related to the compounds we have sputtered: TiN and AIN. Most commonly used solar absorbing material is an inhomogenous composite of small metal particles in a dielectric matrix, i.e. a cermet ${ }^{11}$. The metal and dielectric models are formulated separately. Using the Bruggeman effective medium theory the dielectric function for the inhomogeneous material is obtained. Reflectance and transmittance spectra of modeled thin films on glass substrate can then be calculated and compared to the experimental reflectance and transmittance for a TiAlN film on a transmitting substrate. The dielectric functions of the two components, $\hat{\varepsilon}_{t m}(\omega)$ and $\hat{\varepsilon}_{d}$ contain altogether 12 parameters. These parameters as well as volume fraction and film thickness, are used for fitting. Thus, the optical constants are obtained by fitting the calculated, spectral reflectance and transmittance to the experimental reflectance and 
transmittance over the 0.3-2.5 $\mu \mathrm{m}$ spectral range. This fitting of the calculated R- and T-spectra is an extensive calculation that was simply performed with the software 'CODE' (Coating Designer) ${ }^{12}$, either manually or by choosing "Auto Fitting". To model the interband transitions in TiN, mentioned in the introduction, we have used two broadened oscillator functions. They were originally designed to model the electron-phonon interaction in amorphous semiconductors. ${ }^{13}$ More recent work confirm the usefulness of Brendel oscillators to describe interband in noble-like metals. ${ }^{14}$ They are obtained by convolution of a Drude-Lorentz oscillator with a Gaussian function. The introduction of Brendel oscillators is an option in the CODE package, and we believe that it is a valuable tool for fitting the dielectric function of an inhomogenous material. Our fitting procedure is based on three model equations: The Lorentz-Drude oscillator function with two Brendel oscillators for a transition metal, the dielectric function for $\mathrm{AlON}$ and the Brüggeman effective medium equation:

$$
\begin{gathered}
\hat{\varepsilon}_{t m}(\omega)=\varepsilon_{\infty}+\sum_{i=1}^{2} \frac{1}{\sqrt{2 \pi \sigma_{i}}} \int_{-\infty}^{+\infty} \exp \left(-\frac{\left(x-\omega_{i}\right)^{2}}{2 \sigma_{i}^{2}}\right) \frac{\Omega_{i}^{2}}{\omega_{i}^{2}-x^{2}-i x \Gamma_{i}} d x-\frac{\Omega_{D}^{2}}{\omega^{2}+i \omega \Gamma_{D}} \\
\hat{\varepsilon}_{d}(\omega)=\varepsilon_{1}(\omega)+\varepsilon_{2}(\omega) \\
\delta \frac{\hat{\varepsilon}_{t m}-\hat{\varepsilon}_{e f f}}{\hat{\varepsilon}_{t m}+2 \hat{\varepsilon}_{\text {eff }}}+(1-\delta) \frac{\hat{\varepsilon}_{d}-\hat{\varepsilon}_{\text {eff }}}{\hat{\varepsilon}_{d}+2 \hat{\varepsilon}_{\text {eff }}}=0
\end{gathered}
$$

where $\Omega_{\mathrm{i}}, \omega_{\mathrm{i}}$ and $\Gamma_{\mathrm{i}}$ are the oscillator strengths, the resonances and collision frequencies (or "damping") respectively for the two Brendel oscillators with their Gaussian widths $\sigma_{\mathrm{i}} . \hat{\varepsilon}_{d}$ is the dielectric function for the dielectric and $\delta$ is the volume fraction of the transition metal in the inhomogenous sputtered film. $\hat{\varepsilon}_{\text {eff }}$ finally, is the complex dielectric function for the composite material in the sputtered film. Once $\hat{\varepsilon}_{\text {eff }}$ is calculated, it can be used for ordinary thin film calculations using also the optical constants for the glass substrate.An explicit solution for the convolution integral in (1) is given in the Appendix of ref.13.

In table I, below we list the parameters for the metal part, as obtained after fitting the reflectance- and transmittance-spectra for a semitransparent, sputtered TiAlN-film. The values are given as frequency $(1 / \mathrm{cm})$, wavelength $(\mu \mathrm{m})$ and energy $(\mathrm{eV})$ to simplify the discussion and interpretation. 
Table I: Parameter values for the transition metal model.

\begin{tabular}{|l|l|l|l|}
\hline Oscillators & $1 / \mathrm{cm}$ & $\mu \mathrm{m}$ & $\mathrm{eV}$ \\
\hline$\omega_{1}$ & 27900 & 0.3584 & 3.460 \\
\hline$\Omega_{1}$ & 55240 & 0.1810 & 6.850 \\
\hline$\Gamma_{1}$ & 1.704 & 5869 & $0.2113 \cdot 10^{-3}$ \\
\hline$\sigma_{1}$ & 6431 & 1.555 & 0.7975 \\
\hline$\omega_{2}$ & 4260 & 2.358 & 0.5282 \\
\hline$\Omega_{2}$ & 51540 & 0.1940 & 6.391 \\
\hline$\Gamma_{2}$ & 2450 & 4.081 & 0.3039 \\
\hline$\sigma_{2}$ & 6488 & 1.541 & 0.8045 \\
\hline Conduction electrons & & & \\
\hline$\Omega_{\mathrm{D}}$ & 191.4 & 52.25 & $23.73 \cdot 10^{-3}$ \\
\hline$\Gamma_{\mathrm{D}}$ & 1189 & 8.410 & 0.1475 \\
\hline
\end{tabular}

For the dielectric component we used the dispersive data of AlON obtained from the database of Scout package. The volume-fraction for the transition metal, $\delta$, that was obtained in the fitting was 0.274 .

\section{RESULTS AND DISCUSSION}

Considering the fact that as many as 13 parameters were available for fitting the reflectance and transmittance spectrum for a sputtered film, it is not surprising that good fits of the calculated curves to the experimental points were obtained as shown in Fig 1, below.

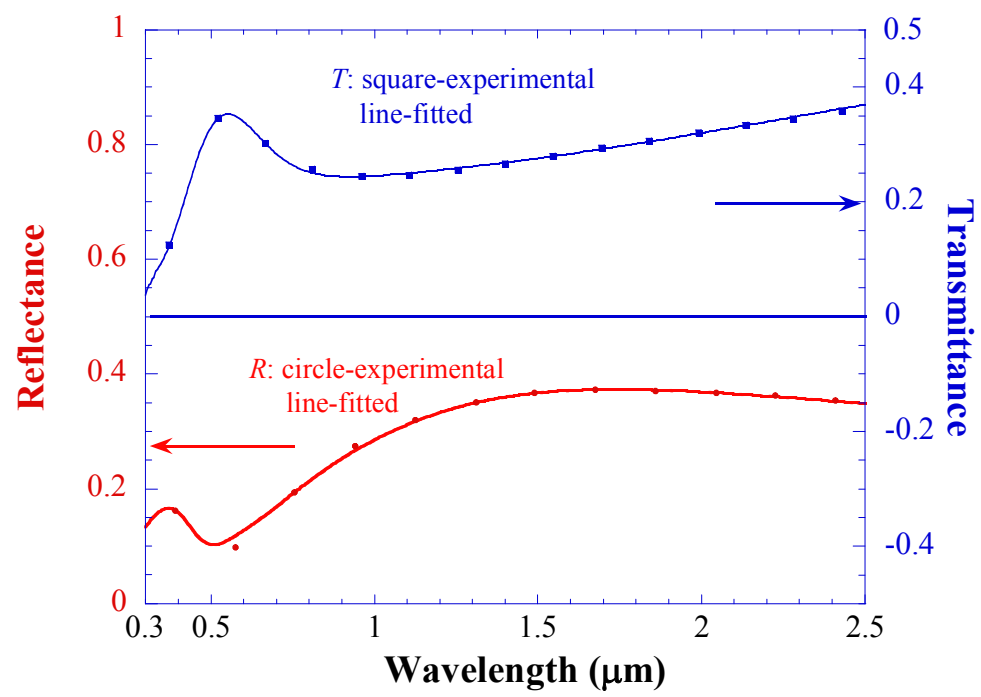

Fitting (lines) of the calculated T-and R-spectra to the experimental points (squares and circles) for the sputtered TiAlN-film on a Corning glass substrate. This fit was obtained with the parameter values in table I. 
In Fig. 2 we reproduce the optical constants $n(\lambda)$ and $k(\lambda)$ that correspond to this TiAlN-model.

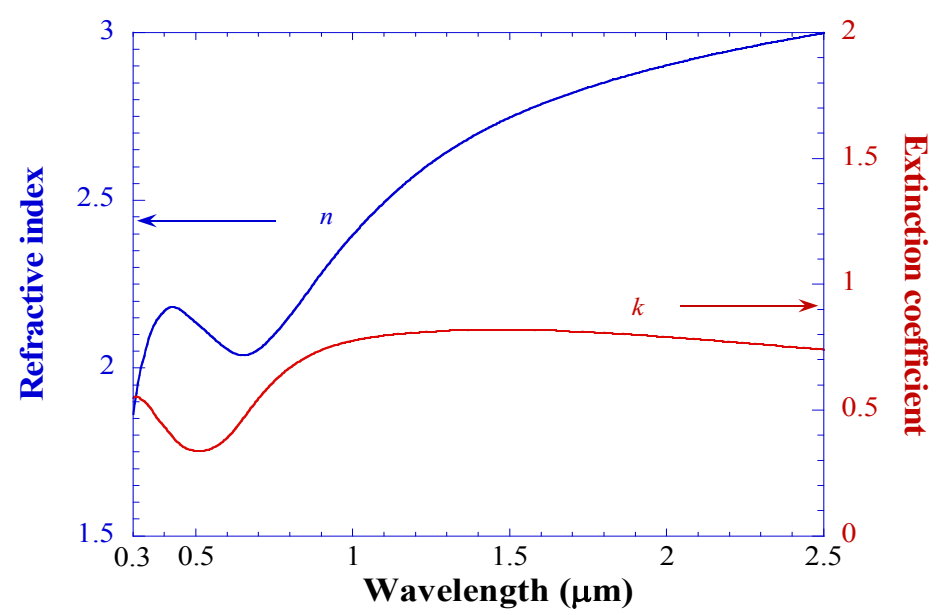

Figure 2 Optical constants $n(\lambda)$ and $k(\lambda)$ as obtained from the parameter values in table I.

One notices that $\mathrm{n}>\mathrm{k}$ over almost the entire spectral region. Optically, our TiAlN-films are thus not metallike. In a separate analysis, the R- and T-data were used to determine the optical absorption with the approximate equation:

$$
\alpha=\frac{1}{d} \ln \left(I_{0} / I\right)=\frac{1}{d} \ln \left(\frac{1-R(\lambda)}{T(\lambda)}\right)
$$

Assuming that the conventional relation for a direct gap in a semiconductor is applicable:

$$
(\alpha h v)^{2}=A\left(h v-E_{g}\right)
$$

We plot the $(\alpha h v)^{2}$-values vs. $h v$ in a standard diagram for extrapolation to $\alpha=0$ to obtain a gap-value. This is shown in Fig. 3 


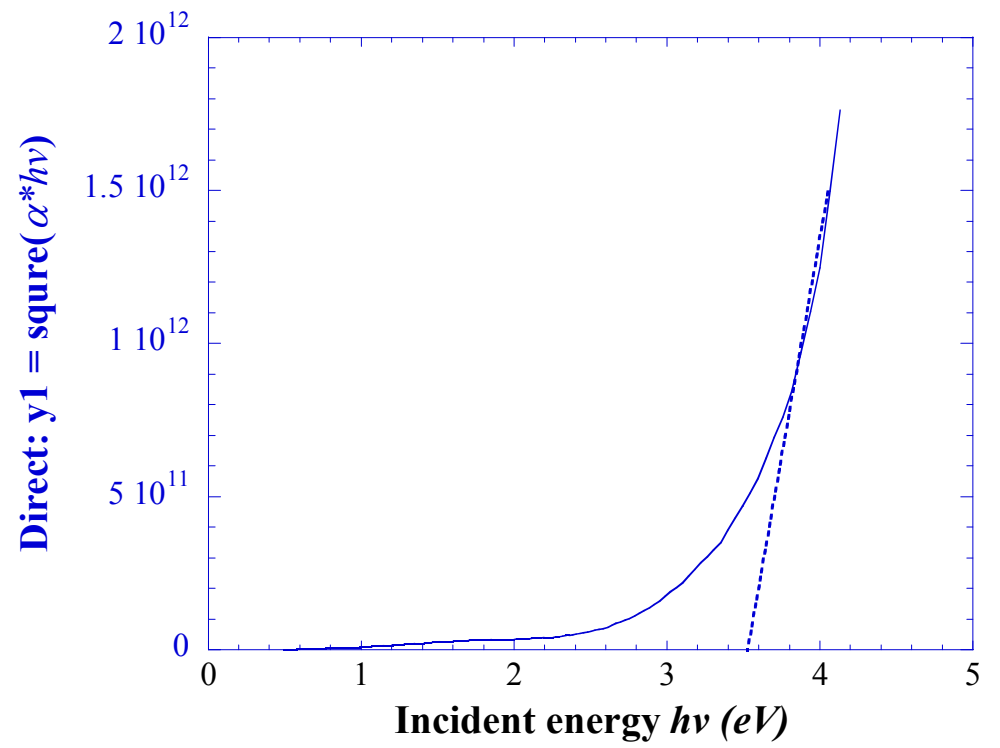

Figure 3 A standard plot of squared absorption in an TiAlN-film as a function of photon energy extrapolated to obtain the value for a direct transition gap.

The curve extrapolates vith the dotted line to $\mathrm{h} v=3.5 \mathrm{eV}$. The agreement with the $\omega_{1}$-value $=3.46 \mathrm{eV}$, in table $\mathrm{I}$, may be fortuitous, but it brings at least out the consistency between the model and the experimental spectra. It also agrees approximately with the visible wavelength where $\mathrm{n} \approx \mathrm{k}$ in Fig. 2, i.e. where $\varepsilon_{1}$ has a zero. Furthermore, we argue that the validity of the model is established by the thickness variation. Once the 12 parameters, corresponding to particular sputtering conditions were established, it could be tested on spectra for the identical film material with different thicknesses. To illustrate the thickness dependence of this model material, we show three calculated reflectance spectra for TiAlN-films on an aluminium substrate in Fig. 4. It should be observed that the model has strong interband absorption centred at $0.36 \mu \mathrm{m}$, i.e. in the visible, and yet the positions of the peaks shift towards longer wavelength with film thickness. The shifts are less than linear with the film thickness, so the similarity with the shift of interference maxima in the case of dielectric films is only qualitative. Linear shifts, as in the dielectric case, should not be expected with the significant k-values for TiAlN. 


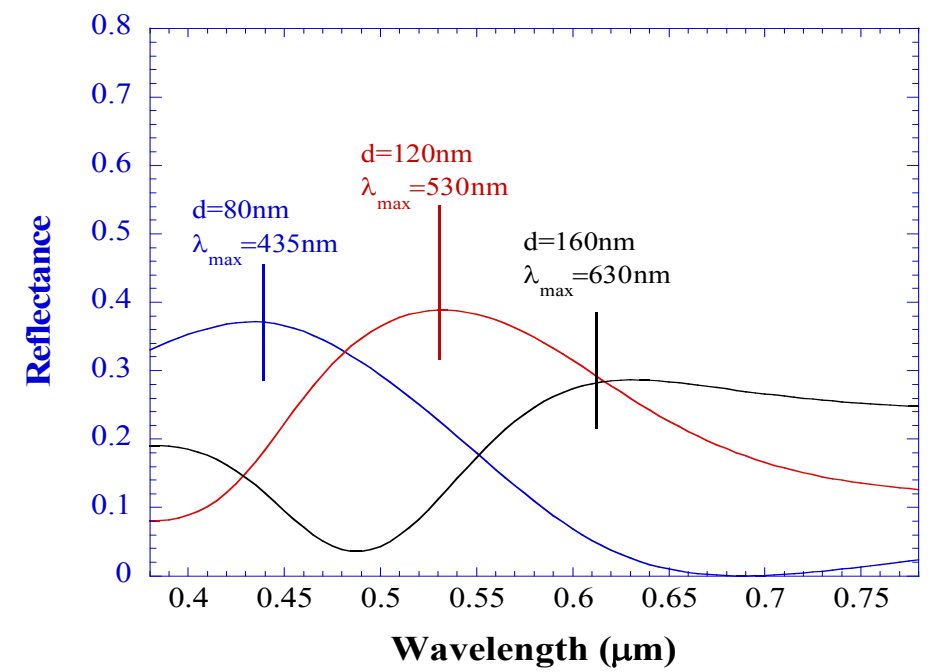

Figure 4 Calculated visible reflectance spectra for modelled TiAlN-films using the parameter values given in Table I and three different thicknesses as indicated.

The peak values in the three calculated spectra are higher than previously reported in the discussion of coloured solar absorbers. ${ }^{3}$ This is because these results are for single TiAlN-films on a metal substrate. No grading or antireflection has been applied. The intention with the model spectra in Fig 4 is to confirm that the model exhibits the peak shifts, i.e. colour change, with film thickness, as previously reported in the solar absorber related works ${ }^{3,4}$.

\section{CONCLUSIONS}

The main purpose of this short contribution is to demonstrate that earlier reports ${ }^{3,4}$ on the potential usefulness of TiAlON-based solar absorbers is in agreement with basic results of well-known thin film optical properties. The experimental observations of colour shifts with film thickness were unexpected, considering that the film is not dielectric. Furthermore, the inhomogenous character of this thin film material is a particular difficulty. It is not possible to model the optical behaviour with only one set of optical constants. The peak in reflectance, giving rise to the colour, has been explained to originate from interband transition in the metal constituent. Optical constants for the film have been determined by fitting model calculations to the experimental R- and T-spectra for films on glass. Using these optical constants in calculations for the TiAlN-film on a metal substrate we have demonstrated that reasonable shifts in the relatively weak visible reflectance do indeed show up as a function of film thickness.

\section{REFERENCES}

1. O. P. Agnihotri, B. K. Gupta Solar Selective Surfaces (J. Wiley, 1981). 
2. G. Wyszecki, Colorimetry, (W.E. Driscoll, W. Vaughan, Ed:s) "Handbook of Optics" OSA, (McGraw-Hill Book Co., N.Y. 1978) Sec. P9, 6-12.

3. D. Zhu, S. Zhao "Chromaticity and optical properties of coloured and black solar-thermal absorber coatings" Solar En. Mat \& Sol. Cells 94, 1630-35 (2010).

4. D. Zhu, F. Mao, S. Zhao "The influence of oxygen in $\mathrm{TiAlO}_{x} \mathrm{~N}_{y}$ on the optical properties of coloured solarabsorbing coatings" In manuscript

5. E.g. in F. Wooten "Optical Properties of Solids" (North Holland 1972).

6. B. Karlsson "Optical Properties of Solids for Solar Energy Conversion" Acta Univ. Upsal.no. 620 (1981).

7. A. Neckel, P. Rastel, R. Eibler, P. Weinberger and K. Schwarz "Results of self-concistent band-structure calculations for ScN, ScO, TiC, TiN, TiO, VC, VN and VO” J. Phys. C: Solid State Phys. 9 , 579 (1976).

8. A. Delin, O. Eriksson, R. Ahuja, B. Johansson, M.S.S. Brooks, T. Gasche, S. Auluck, J. M. Wills "Optical

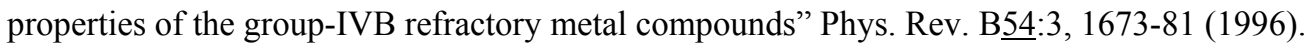

9. See e.g. http://www.pvd-coatings.co.uk/coatings/ceramic-coatings/tialn-coating/

10. M. Brogren, G. L. Harding, R. Karmhag, C. G. Ribbing, G. A. Niklasson, L. Stenmark "Titaniumaluminium-nitride coatings for satellite temperature control” Thin Solid Films $\underline{370}, 268$ (2000).

11. R. A. Buhrman, H. G. Craighead "Composite Film Selective-Absorbers" in Solar Materials Science (Ed. L.E. Murr, Acad. Press, N.Y. 1980), 277-92.

12. M. Theiss, Hard and software for optical spectroscopy ( Dr Bernhard-Klein-Str. 110, D-52078 Aachen, Germany 2002).

13. R. Brendel, D. Borman "An infrared dielectric function model for amourphous solids" J. Appl. Phys. $\underline{71}$, 1-6 (1992).

14. A. D. Rakic, A. B. Djurišic, J. M. Elazar, M. L. Majewski ” Optical Properties of Metallic Films for VerticalCavity Optoelectronic Devices” Appl. Opt. 37: 22, 5271-5283 (1998). 